\title{
Improving Localization of Cardiac Geometry Using ECGI
}

\author{
Jake A Bergquist ${ }^{1,2,3}$, Jaume Coll-Font ${ }^{4}$, Brian Zenger ${ }^{1,2,3,5}$, Lindsay C Rupp ${ }^{1,2,3}$, Wilson W \\ Good $^{1,2,3}$, Dana H Brooks6, Rob S MacLeod ${ }^{1,2,3}$ \\ ${ }^{1}$ Scientific Computing and Imaging Institute, University of Utah, SLC, UT, USA \\ ${ }^{2}$ Nora Eccles Cardiovascular Research and Training Institute, University of Utah, SLC, UT, USA \\ ${ }^{3}$ Department of Biomedical Engineering, University of Utah, SLC, UT, USA \\ ${ }^{4}$ Cardiovascular Bioengineering \& Imaging (CBM) Lab at the Massachusetts General Hospital, \\ Boston (MA) and Harvard Medical School, Boston, MA, USA \\ ${ }^{5}$ School of Medicine, University of Utah, SLC, UT, USA \\ ${ }^{6}$ Department of Electrical and Computer Engineering, Northeastern University, Boston, MA, USA
}

\begin{abstract}
Introduction: Electrocardiographic imaging (ECGI) requires a model of the torso, and inaccuracy in the position of the heart is a known source of error. We previously presented a method to localize the heart when body and heart surface potentials are known. The goal of this study is to extend this approach to only use body surface potentials.

Methods: We used an iterative coordinate descent optimization to estimate the positions of the heart for several consecutive heartbeats relying on the assumption that the epicardial potential sequence is the same in each beat. The method was tested with data synthesized using measurements from a isolated-heart, torso-tank preparation. Improvement was evaluated in terms of both heart localization and ECGI accuracy.

Results: The geometric correction resulted in cardiac geometries closely matching ground truth geometry. ECGI accuracy increased dramatically by all metrics using the corrected geometry.

Discussion: Future studies will employ more realistic animal models and then human subjects. Success could impact clinical ECGI by reducing errors from respiratory movement and perhaps decrease imaging requirements, reducing both cost and logistical difficulty of ECGI, widening clinical applicability.
\end{abstract}

\section{Introduction}

Electrocardiographic imaging (ECGI) is a tool for the noninvasive assessment of cardiac electrical activity using body surface potential (BSP) signals that has been applied clinically to successfully diagnose a number of cardiac dysfunctions.[1] ECGI, like many imaging modali- ties, leverages the biophysics that dictate how source signals project to measurement locations (solving a'forward' problem) and uses this relationship to solve for the reverse, i.e., estimate features of the source, given remote measurements (solving an 'inverse' problem).[2] The forward problem in electrocardiography depends on the geometry of the torso and organs and their conductivities. Solutions to this forward problem are known to be well behaved in the sense that small errors in sources or models tend to result in small effects on the solution. On the other hand, the associate inverse problems are ill-posed, i.e., small errors in the inputs, such as errors in the position of the heart, have a significant impact on the accuracy of solutions.[3] Hence, constructing an accurate forward model is critical to enabling an accurate ECGI solution. However, errors in cardiac location remain a mostly unaddressed source of error for both clinical and experimental uses of ECGI.[4] The position of the heart changes during respiration and due to shifts in position of the patient. These errors cannot be easily addressed with improved medical imaging or segmentation and there is little expectation of addressing them using procedural changes, such as requiring the patient to maintain breath holds. Thus directly addressing this source of error could improve the accuracy of all ECGI implementations, both clinical and experimental.

There have been a number of research efforts to address the cardiac localization error problems. These approaches seek to leverage properties of ECGI such as changes to the L-curve of the ECGI inverse problem with changes in geometry, or differences between simulated and measured BSPs.[5] Our group previously localized the heart using an optimization based approach that assumed availability of potentials on both the body surface (BSPs) and the heart surface (electrograms, EGMs).[6,7] The cardiac position was found by optimizing over a set of position pa- 
rameters to minimize the residual error between measured BSPs and those simulated using the cardiac forward problem. $[6,7]$ In this study, we addressed the scenario in which only BSPs from several heartbeats and a nominal geometry are available, with the goal of correcting the cardiac geometry using entirely non-invasive measurement techniques. We quantified the results of our reconstructions, both in terms of the cardiac position and ECGI solutions.

\section{Methods}

Our geometric correction framework for cardiac position is divided into two alternating main steps that are iteratively repeated: 1) estimating a new cardiac position assuming a candidate set of inverse EGMs (iEGMs), and 2) estimating new iEGMs assuming a candidate cardiac position.

Estimating Cardiac Position: We parameterized the location of the heart and estimated the correct position using a forward problem based optimization described by Coll-Font et al..[6] Briefly, the heart is assumed to be a rigid body and its position within the torso is parametrized by 6 degrees of freedom: $x, y$, and $z$ position of the centroid of the heart, and pitch (side to side swing), yaw (rotation of the septal axis about vertical axis of the torso), and roll (rotation about the septal axis). Given a set of candidate iEGMs, we used an interior point trust region optimization implemented in MATLAB to estimate a new position of the heart by minimizing a cost function whose value was the root mean squared error (RMSE) between measured and forward computed BSPs. The forward computed BSPs were generated using the boundary element method (BEM) for extracellular potentials.

Estimating inverse EGMs: Given a candidate set of position and orientation parameters, we used a standard ECGI method, with Tikhonov 2nd order regularization to estimate iEGMs. However, if we used a single cardiac geometry and BSPs from a single heart beat then the resulting position estimate would be to not move the heart at all. To avoid this trivial solution we used a sequence of BSPs from multiple heartbeats to estimate iEGMs for one beat, with the assumption that any differences between beats in the recorded BSPs are the result of differences in cardiac position, and that the source EGMs are the same for each beat. As a consequence, in the Estimating Cardiac Position step we can then use the beat-to-beat variations in BSPs and the estimated iEGMs to optimize for the cardiac position for each heartbeat.

To summarize, the algorithm iteratively repeats the cardiac position and iEGMs estimation steps to determine the cardiac position for each heartbeat. The full algorithm is described in Algorithm 1.

Dataset: To evaluate this method, we generated a syn-

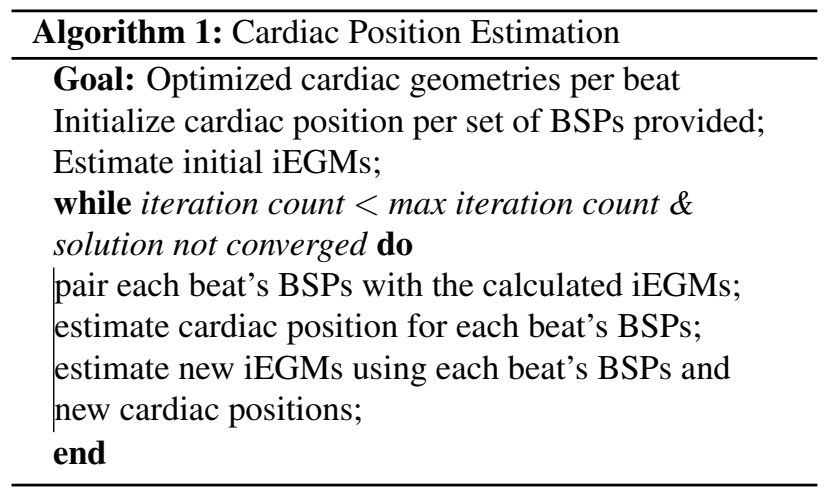

thetic dataset in which EGMs, BSPs and cardiac position were known for a set of 100 heartbeats. The EGMs and basic geometric data came from an isolated-heart and torso tank preparation described previously.[8,9] Briefly, an isolated heart was suspended within a torso-shaped tank filled with electrolyte solution and 192 embedded electrodes in contact with the solution. A rigid pericardiac cage recording array equipped with 256 silver-silver chloride electrodes enclosed the isolated heart. Signals were recorded continuously from the electrode arrays at $1 \mathrm{kHz}$ using a custom acquisition system during a variety of interventions including ventricular pacing. Signals were filtered, baseline corrected, and segmented using PFEIFER, an opensource signal processing tool.[10] Cage and torso electrode positions were recorded using a mechanical digitizer.

We selected a single left ventricularly paced heart beat and used the EGMs recorded from the 256 electrodes of the pericardiac cage as ground truth EGMs. We generated 100 sets of BSPs from these EGM recordings by numerically moving the cardiac cage within the torso geometry, calculating a BEM solution, and adding Gaussian noise at an SNR of 30dB. The parameters of the cardiac positions for each BSP are shown in Figure 1(black diamonds). The nominal position of the cage corresponded to its digitized position. During optimization, the cardiac geometries for all 100 beats were initialized to this nominal position at Pitch $=$ Yaw $=$ Roll $=X=Y=Z=0$, which we subsequently considered the 'unoptimized' position.

Analysis: We measured the success of our geometric correction framework by both its ability to reconstruct the cardiac position as well as its ability to improve the estimation of iEGMs as compared to the unoptimized position. Position reconstruction accuracy was assessed according to two metrics: mean per-electrode distance, and centroid distance. Mean per-electrode distance is the average Euclidean distance between electrode positions in the ground truth and optimized cardiac geometries. Centroid distance is the distance between the centroids of the ground truth and optimized cardiac geometries. We also assessed the 
position reconstruction qualitatively by comparing ground truth to optimized locations.

We quantified differences in ECGI solutions before and after geometry optimization and compared these solutions to solutions obtained using the ground truth cardiac position. For each of the 100 beats, the cardiac geometry was placed at either the unoptimized, optimized, or ground truth positions. Forward matrices were computed with BEM, and iEGMs were estimated with an inverse solver. We selected the Tikhonov 2nd order regularization parameter using the Frobenius norm (across an entire beat) Lcurve method.[2]. We quantified differences in the iEGMs using spatial correlation (SC) averaged over all time instances, temporal correlation (TC) averaged over all electrodes, the root mean squared error (RMSE), as well as carrying out qualitative visual examination of results.[8]

\section{Results}

Geometric Reconstruction: Our geometric optimization framework was able to reconstruct the cardiac position with high fidelity. The 6 position parameter reconstructions (Figure 1 red circles) generally agreed closely with the ground truth parameters (black diamonds), especially for the rotation parameters. The mean per-electrode distance averaged over all 100 reconstructed positions was $8.50 \mathrm{~mm}$ with a standard deviation of $0.12 \mathrm{~mm}$, and the average centroid distance was $6.43 \mathrm{~mm}$ with a standard deviation of $0.56 \mathrm{~mm}$. Qualitative assessment suggested a constant small offset between ground truth and optimized geometries for all 100 beats.

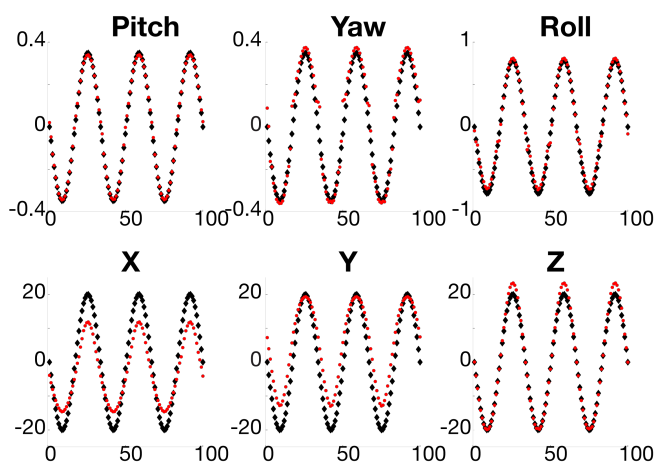

Figure 1. Ground truth (black diamonds) and reconstructed (red dots) cardiac position parameters for each parameterized degree of freedom. Pitch, yaw, and roll are defined (in radians) about a septal axis, $\mathrm{x}, \mathrm{y}$, and $\mathrm{z}$ translations are in mm's.

ECGI Improvement:Geometry optimization resulted in more accurate inverse solutions as compared to using the unoptimized geometries. The metrics shown in Table 1 confirm that the optimization resulted in inverse solutions that closely resembled the solutions obtained using the ground truth positions. Figure 2 shows a compar-

\begin{tabular}{|c|c|c|c|}
\hline & SC & TC & RMSE \\
\hline Pt & $0.96 \pm 0.001$ & $0.96 \pm 0.002$ & $0.24 \pm 0.006$ \\
Popt & $0.88 \pm 0.002$ & $0.93 \pm 0.003$ & $0.31 \pm 0.005$ \\
P0 & $0.52 \pm 0.27$ & $0.51 \pm 0.27$ & $1.14 \pm 0.58$ \\
\hline
\end{tabular}

Table 1. ECGI solution accuracy metrics for different cardiac locations. P0 uses the nominal unoptimized cardiac position, Pt uses the ground truth cardiac positions, and Popt uses the optimized positions. RMSE is in millivolts, spatial correlation (SC) and temporal correlation (TC) are unitless.

ison between the inverse solutions using unoptimized, optimized, and true positions. We see that even in the worst case scenario, as defined by minimum spatial correlation, the optimized geometry resulted in better inverse reconstructions than the unoptimized geometry.

\section{Discussion and Conclusions}

The results presented here support the hypothesis that our algorithm is able to reconstruct the cardiac position using BSPs and that these positions improve the inverse reconstructions of the EGMs. This method reliably corrected the cardiac geometry in our synthetic dataset to within an average of $8 \mathrm{~mm}$ (based on per-electrode distance) and resulted in improvements in the inverse reconstruction over the unoptimized positions seen in Table 1.

The reconstruction accuracy varied for each parameter, with pitch, yaw, roll, and vertical (z) position showing noticeably better agreement with the ground truth parameters than $\mathrm{x}$ and $\mathrm{y}$ directions (Figure 1). We notice that poorer reconstruction tended to occur at the extrema of the range of motion (and for yaw also around the center of the range). We speculate that the difficulty in reconstruction yaw values near zero may be due to a singularity in the coordinate system resulting in similarity of yaw to roll rotations when pitch is also near zero. However, despite this unresolved error, we still observed that optimized cardiac positions, measured with per-electrode-distance, were closer to the ground truth than the unoptimized positions. The optimization not only improved the accuracy of the ECGI solutions but also dramatically decreased the variability in ECGI accuracy for all measured statistics (as assessed by the reduced standard deviations after optimization). The standard deviations of the accuracy metrics after optimization were comparable to the standard deviations found using the true cardiac positions. The remaining discrepancy between the ECGI solutions of the optimized and true cardiac geometries is likely due to the residual geometric error that our algorithm was unable to correct.

This study was limited by the use of a synthetic dataset. The simulation of simple, in-phase periodic variation of the 6 parameters may not be realistic. The dynamic range may not be adequate to describe motion in patients. There 

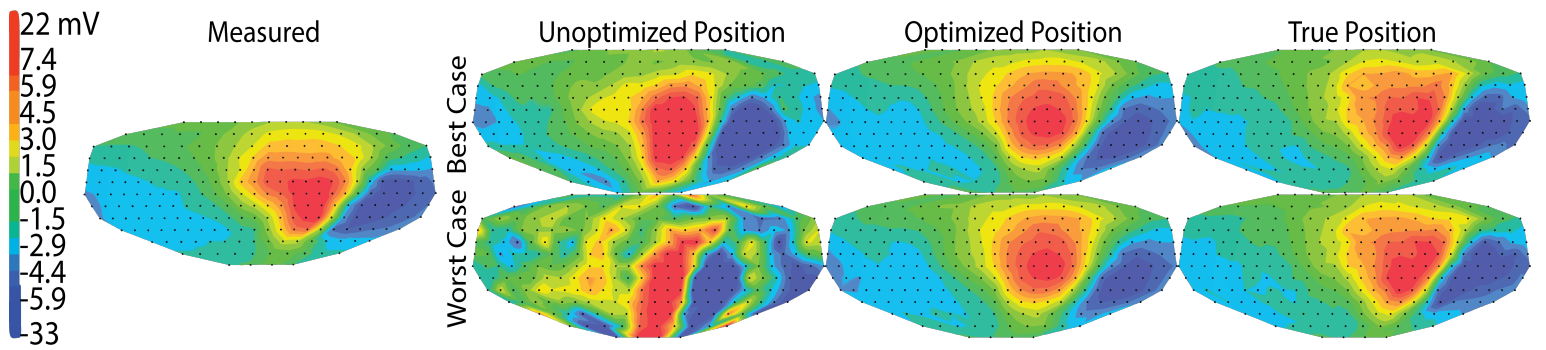

Figure 2. Identified best and worst case potential reconstructions visualized on the cardiac geometry. Values displayed are in millivolts. The cardiac geometry was flattened for visualization, and a time point at the peak of the QRS was selected for visualization. The first column shows the ground truth measured EGM. The second through fourth columns show the inverse reconstructions using Unoptimized, Optimized, and True cardiac positions respectively. Best case reconstruction (top row, $\mathrm{SC}=0.88$ ) and the worst case reconstruction (bottom row, $\mathrm{SC}=0.87$ ) were based on inverse solutions found using optimized geometries.

also remains some geometric error, suggesting room for improvement in the optimization. However, the ability of the algorithm to reduce geometric error and improve ECGI solution accuracy demonstrates proof of concept. Our next steps will be to use more realistic synthetic and experimental datasets. We expect that including temporal beatto-beat constraints such as assuring that positions of neighboring beats are similar or allowing for a fitting of a respiratory cycle may improve the accuracy of reconstruction for respiratory motion. We predict that our method will be able to improve the accuracy of ECGI solutions in all contexts in which the cardiac geometry is moving relative to the torso, a scenario common in both clinical and experimental settings. We also theorize that this same method could be used to correct for other errors in the geometric model such as conductivity values in an inhomogenous torso model. The development of this geometric correction method may lead to additional benefits such as utilizing less accurate but also less expensive imaging modalities such as echocardiography to capture the geometric model needed for ECGI, or even allow the use of template cardiac geometries to replace patient specific imaging.

\section{Acknowledgments}

Support for this research came from the NIHNIGMS Center for Integrative Biomedical Computing (www.sci.utah.edu/cibc), NIH NIGMS grants P41 GM103545 and R24 GM136986, and the Nora Eccles Treadwell Foundation for Cardiovascular Research.

\section{References}

[1] Cluitmans M, Brooks D, MacLeod R, Doessel O, Guillem M, Dam PV, Svehlikova J, He B, Sapp J, Wang L, Bear L. Consensus on validation and opportunities of electrocardiographic imaging: From technical achievements to clinical applications. Front Physiol September 2018;9(1305):1-19.

[2] Milanic M, Jazbinsek V, Macleod R, Brooks D, Hren R.
Assessment of regularization techniques for electrocardiographic imaging. J Electrocardiol Jan.-Feb. 2014;47(1):2028.

[3] Swenson D, Geneser S, Stinstra J, Kirby R, MacLeod R. Cardiac position sensitivity study in the electrocardiographic forward problem using stochastic collocation and boundary element methods. Ann Biomed Eng December 2011;39(12):2900-2910.

[4] MacLeod R, Ni Q, Punske B, Ershler P, Yilmaz B, Taccardi. B. Effects of heart position on the body-surface ECG. J Electrocardiol Feb. 2000;33((supp)):229-238.

[5] Rodrigo M, Climent AM, Liberos A, Hernandez-Romero I, Arenal A, Bermejo J, Fernandez-Aviles F, Atienza F, Guillem MS. Solving inaccuracies in anatomical models for electrocardiographic inverse problem resolution by maximizing reconstruction quality. IEEE Transactions on Medical Imaging 2018;37(3):733-740.

[6] Coll-Font J, Brooks DH. Tracking the position of the heart from body surface potential maps and electrograms. Frontiers in Physiology 2018;9:1727.

[7] Coll-Font J, Brooks DH. Ecg-based reconstruction of heart position and orientation with bayesian optimization. In Computing in Cardiology Conference (CinC), 2017, volume 44. IEEE, 2017; 1727.

[8] Bergquist J, Good W, Zenger B, Tate J, MacLeod R. Optimizing the reconstruction of cardiac potentials using a novel high resolution pericardiac cage. In Computing in Cardiology 2019, volume 46. 2019; 1-4.

[9] Bergquist JA, Good WW, Zenger B, Tate JD, MacLeod RS. The Cardiac Forward Problem: A Benchmark Study. In Preparation 2020;

[10] Rodenhauser A, Good W, Zenger B, Tate J, Aras K, Burton B, MacLeod R. PFEIFER: Preprocessing framework for electrograms intermittently fiducialized from experimental recordings. J Open Source Software 2018;3(21):472.

Address for correspondence:

Jake Bergquist

University of Utah

72 Central Campus Dr, Salt Lake City, UT 84112

jbergquist@sci.utah.edu 